\title{
Nurses can play a crucial role in the recognition of primary immune deficiency
}

\author{
Esther de Vries \\ Department of Paediatrics, Jeroen Bosch Hospital, 's-Hertogenbosch, the Netherlands
}

Correspondence: Dr. Esther de Vries. Address: Department of Paediatrics, Jeroen Bosch Hospital, 's-Hertogenbosch, the Netherlands. Email: e.d.vries@jbz.nl

Received: September 18, 2012

Accepted: December 24, 2012

Online Published: August 16, 2013

DOI : $10.5430 /$ jnep.v4n1p96

URL: http://dx.doi.org/10.5430/jnep.v4n1p96

\section{Abstract}

Primary immunodeficiency diseases are disorders of immunological functioning leading to infections that are too frequent, too severe, and too resistant to treatment. Also, other manifestations such as unusual forms of autoimmunity and lymphoproliferation may be present. Timely recognition of these diseases is important for the patient's prognosis, and for genetic counselling of the family. Nurses can play a crucial role in the timely recognition of patients with these disorders. In this paper, the potential for recognition of primary immunodeficiency diseases (PIDs) s by nurses is discussed, and some examples of PID such as common variable immunodeficiency (CVID) and severe combined immunodeficiency (SCID) are presented.

\section{Key words}

Primary immunodeficiency disease, Antibody deficiency, Recognition

\section{I ntroduction}

Classical primary immunodeficiency disease (PID) ${ }^{[1-4]}$ is relatively rare, approximately 1:500 to 1:500 000 in the general population, depending on the type of the disease. In PID, the immune system intrinsically fails in its functions, either partially or completely. In general, these diseases are genetically determined, either directly by a disease-causing mutation, or indirectly because the genetic make-up enables environmental factors to cause the disease in a multi-factorial design. The "typical" PID patient suffers from infections: too many, too serious, too resistant to therapy. However, other manifestations can be present as well, such as autoimmune disease, failure-to-thrive and malignancy ${ }^{[5]}$. Early detection of PID, before serious infections have compromised the patient's general condition, is important for prognosis, and enables timely genetic counselling of the family ${ }^{[6]}$. It can be difficult to identify PID patients, especially for non-immunologists. Therefore, a diagnostic protocol was published in cooperation with the Clinical Working Party of the European Society for Immunodeficiency in Clinical and Experimental Immunology in 2006 (first edition) and 2011 (update) ${ }^{[1,2]}$.

Nurses can play a crucial role in the early detection of PIDs, by giving out an alert when they notice suggestive signals in their patients. This paper is based upon the aforementioned protocol, which can help them in this task. Although many PIDs present in childhood, the most common clinically significant PID, common variable immunodeficiency (CVID, see below), has a peak onset in the second and third decades of life ${ }^{[7]}$. Therefore, nurses working with adults as well as children could play a role. 


\section{Picking up the signs of PID: it can be life saving}

Considering the possibility of a PID is the key to the diagnosis. It all starts from the clinical presentation of the patient, and fortunately immunological expertise is not needed for this first step. The clinical presentations can be observed by any health care professional.

Infections are the hallmark of immunodeficiency ${ }^{[6]}$, but most patients with infections do not have PID. Therefore, efficient identification of PID within the large pool of potential cases is difficult. However, some simple rules help to separate those patients in whom PID is more likely to be present than in others. In Table 1, a list of "suspicious" infectious situations is given ${ }^{[1,2]}$. The most common presentation of PID is with recurrent respiratory infections, however. Confusingly, this clinical presentation is often encountered in everyday practice, especially in young children, but also in older children and adults in any pulmonology or ear-nose-throat (ENT) service. Most of these patients do not have PID. However, when more than one pneumonia occurs, bronchiectasis is present, the infections fail to clear with conventional treatment, or continue to occur when a young child gets older, immunological investigations are needed, and consultation of an immunologist is highly recommended. Timely recognition of PID is important, as successful treatment of a child with severe PID such as severe combined immunodeficiency (SCID, see below) is dependent on rapid recognition ${ }^{[8]}$. Less urgent, but still important if future organ damage and decreased quality of life and life-span are to be prevented, is the timely recognition of late-onset as well as less pronounced forms of PID in older children and adults ${ }^{[7]}$.

Table 1. Infectious Situations That Should Alert to Potential PID

- Recurrent (proven) bacterial infections

- Severe infections (e.g. meningitis, osteomyelitis, pneumonia), especially $>1$

- Infections that present atypically, are unusually severe or chronic or fail regular treatment

- Infections caused by an unexpected or opportunistic pathogen

- Severe or long-lasting warts, generalized mollusca contagiosa

- Extensive candidiasis

- Complications of vaccination [disseminated bacille Calmette-Guérin (BCG) or vaccinia infection, paralytic polio]

- Abscesses of internal organs; recurrent subcutaneous abscesses

- Prolonged or recurrent diarrhoea

Note. PID, primary immunodeficiency disease

Other symptoms than infections may be more prominent at first, such as the failure-to-thrive PIDs may cause in children, autoimmune manifestations, especially in adults, as well as unusual lymphoid or granulomatous diseases. Unusual autoimmune disease, atypical Crohn disease, an unexpected course in lymphoproliferative disease, all these may be caused by PID, and warrant further immunological investigation (see Table 2). Nurses can give the alert for potential PID in these “atypical” patients ${ }^{[5]}$.

\section{PI Ds are not so rare when they run in the family}

Family history is a vital clue to the diagnosis of PID, as although patients with recurrent infections do not often have PID, this becomes much more likely when it "runs in the family". This also holds true for adult patients who can present with late-onset forms of disease. It is not always easy to discern when PID runs in the family; the formal diagnosis has not always been made. Familial occurrence of similar symptoms, unexplained early infant deaths, or deaths due to infection in the developed world could give a clue. Also, consanguinity -either known or suspected - and uncommonly large numbers of family members with autoimmune disease or haematological malignancy may direct towards PID, especially if 
recurrent infections were also present in those family members. Nurses can explore this issue when talking to a patient about the family history.

Table 2. Useful Laboratory Tests to Screen for PID

\begin{tabular}{ll}
\hline Test & Can detect \\
\hline Blood count and differential & $\begin{array}{l}\text { Granulocytopenia, lymphocytopenia, thrombocytopenia, anaemia } \\
\text { IgA-deficiency, hypogammaglobulinemia, agammaglobulinemia, } \\
\text { hyper-IgE syndrome }\end{array}$ \\
$\begin{array}{l}\text { Immunoglobulin isotypes (IgG, IgA, IgM; IgE) } \\
\text { IgG-subclasses (IgG1, IgG2, IgG3, IgG4) }\end{array}$ & $\begin{array}{l}\text { Deficiencies of specific antibody production, anti-polysaccharide } \\
\text { antibody deficiency }\end{array}$ \\
$\begin{array}{l}\text { Specific antibody response to vaccination (tetanus, } \\
\text { pneumococcal polysaccharide) }\end{array}$ & $\begin{array}{l}\text { Malignancy (e.g. Waldenström) } \\
\text { M-protein (monoclonal immunoglobulin) }\end{array}$ \\
CH50 and AP50 & $\begin{array}{l}\text { HIV-infection, AIDS (differential diagnosis of PIDs, especially } \\
\text { important in endemic areas) }\end{array}$ \\
HIV-serology and PCR & $\begin{array}{l}\text { Absence of B-lymphocytes, T-lymphocytes, NK-cells and/or subsets } \\
\text { thereof }\end{array}$ \\
Lymphocyte subpopulations by flowcytometry & Known and as yet unknown PID-mutations \\
\hline Genetic analysis &
\end{tabular}

Note. This table is limited to items useful in diagnosing PIDs and their complications. Ig, immunoglobulin; PID, primary immunodeficiency disease.

\section{PI Ds and HIV/ AI DS are two different things entirely}

PIDs are often confused with human immunodeficiency virus (HIV)-infection and acquired immunodeficiency syndrome (AIDS), because the symptoms of failing immunity are similar. However, PIDs are genetically or at least multifactorially determined, whereas AIDS is a secondary phenomenon, the result of infection with a virus, HIV. Also, drugs, malignancies, and diseases which cause protein and/or lymphocyte loss may cause secondary immunodeficiency; this is more common than unrecognized PID in adults ${ }^{[9]}$. It is important to remember these possibilities as well.

\section{Some examples of PID}

Developments in the field of PIDs have been very rapid in the past decade, and over 200 diseases have now been recognized. However, some are much more frequent than others, and nurses are more likely to encounter those. Also, even though a nurse has no knowledge about a specifically encountered PID type, the recognition of potential immunedeficiency as such is enough to start the search for an underlying PID in suggestive cases. By consulting an immunedeficiency specialist in such a case, a nurse can make the difference to the patient in question, even without knowing exactly what type of PID might be present.

Predominantly antibody deficiencies (PADs) are the most common forms of PIDs; in these cases, the amount of antibodies or immunoglobulins is too low or they are totally absent ${ }^{[5]}$. Antibodies mainly protect against bacterial infections, they also contain memory against microbes that have invaded the body in the past. Immunisation programs are based upon this capacity of the immune system. Patients with PAD mainly suffer from recurrent pulmonary and ENT infections, but may also have autoimmune disease, lymphadenopathy, splenomegaly and/or hematological malignancy, especially when they are adults suffering from the most common form of PAD, CVID ${ }^{[7]}$. In CVID, B-lymphocytes, the white blood cells from which immunoglobulins originate, are present but they don't function properly, and immunoglobulin levels are (very) low. In CVID-like PAD-patients, the immunoglobulin levels are less abnormal, but responses to immunisation are insufficient; their pulmonary problems may be just as severe, however, as in "true" CVID ${ }^{[10]}$. In much rarer forms of PAD that are caused by a mutation in a single gene, B-lymphocytes are absent in the blood. The treatment of PAD-patients consists of prompt antibiotic treatment of infections, antibiotic prophylaxis and/or lifelong immunoglobulin substitution therapy by the intravenous or subcutaneous route, depending on their clinical situation. 
SCID is the most severe and acute life-threatening form of PID that requires immediate specialised care and intensive treatment by stem-cell transplantation, or - experimentally - gene therapy to enable survival beyond the first year of life ${ }^{[8]}$. Children with SCID often present with failure-to-thrive, intractable diarrhea and/or unusual, severe infections.

Several PIDs present with typical dysmorphic features, and in many eponymous syndromes that have been well-known to paediatricians for a long time immunological abnormalities have been shown to be present when looked for ${ }^{[11]}$. In these cases, the dysmorphic features may help to classify the patient in the right diagnosis category. Also, correction of e.g. antibody deficiency by immunoglobulin substitution may decrease the number of pulmonary infections in retarded syndromic children and thereby increase their quality of life, even when their anatomical and functional reasons for pulmonary infection inherent to the syndrome continue to exist.

\section{Conclusion}

PIDs should be considered in patients with recurrent or severe infections, because early diagnosis and treatment can reduce mortality and morbidity. Nurses can play a crucial role in this, whether they care for children or adults, and irrespective of their own degree of immunological knowledge. The key to the diagnosis of a PID is to consider the possibility of a PID being present. Nurses that recognize the most common clinical presentations can - and hopefully will - make the difference!

\section{References}

[1] De Vries E, for the Clinical Working Party of the European Society for Immunodeficiencies (ESID). Patient-centred screening for primary immunodeficiency: a multi-stage diagnostic protocol designed for non-immunologists. Clin Exp Immunol. 2006; 145: 204-14. PMid:16879238 http://dx.doi.org/10.1111/j.1365-2249.2006.03138.x

[2] De Vries E, in collaboration with European Society for Immunodeficiencies (ESID) members. Patient-centred screening for primary immunodeficiency, a multi-stage diagnostic protocol designed for non-immunologists: 2011 update. Clin Exp Immunol. 2011; 167: 108-19. PMid:22132890 http://dx.doi.org/10.1111/j.1365-2249.2011.04461.x

[3] Fischer A. Human primary immunodeficiency diseases. Immunity. 2007; 27:835-45. PMid:18093537 http://dx.doi.org/10.1016/j.immuni.2007.11.012

[4] Boyle JM, Buckley RH. Population prevalence of diagnosed primary immunodeficiency diseases in the United States. J Clin Immunol. 2007; 27: 497-502. PMid:17577648 http://dx.doi.org/10.1007/s10875-007-9103-1

[5] Al-Herz W, Bousfiha A, Casanova JL, et al. Primary immunodeficiency diseases: an update on the classification from the International Union of Immunological Societies Expert Committee for Primary Immunodeficiency. Frontiers Immunol. 2011; 2: 54.

[6] Stiehm ER, Chin TW, Haas A, Peerless AG. Infectious complications of the primary immunodeficiencies. Clin Immunol Immunopathol. 1986; 40: 69-86. http://dx.doi.org/10.1016/0090-1229(86)90070-X

[7] Lucas M, Lee M, Lortan J, Lopez-Granados E,Misbah S, Chapel H. Infection outcomes in patients with common variable immunodeficiency disorders: relationship to immunoglobulin therapy over 22 years. J Allergy Clin Immunol. 2010; 125: 1354-60. PMid:20471071 http://dx.doi.org/10.1016/j.jaci.2010.02.040

[8] Rosen FS. Severe combined immunodeficiency: a pediatric emergency. J Pediatr. 1997; 130: 345-6. PMid:9063405

[9] Azar AE, Ballas ZK. Evaluation of the adult with suspected immunodeficiency. Am J Med. 2007; 120: 764-8. PMid:17765042 http://dx.doi.org/10.1016/j.amjmed.2006.12.013

[10] Van de Ven AA, van Montfrans JM, Terheggen-Lagro SW, et al. A CT scan score for the assessment of lung disease in children with common variable immunodeficiency disorders. Chest. 2010; 138: 371-9. PMid:20299624 http://dx.doi.org/10.1378/chest.09-2398

[11] Ming JE, Stiehm ER, Graham JM Jr. Syndromes associated with immunodeficiency. Adv Pediatr. 1999; 46: $271-351$. PMid:10645468 